\title{
Modelos matemáticos de previsão aplicados à gestão hídrica
}

O avanço da urbanização e a ausência de políticas públicas eficazes se apresentam como desafios para a disposição adequada dos serviços de abastecimento hídrico, entretanto, no Brasil, a tomada de consciência sobre a relevância da preservação de tal recurso encontra como barreira sua abundância. Uma alternativa para auxiliar atividades de gestão dos recursos hídricos é a utilização de modelos matemáticos. Este trabalho tem por objetivo elaborar um modelo matemático de previsão do consumo hídrico tomando por base o proposto por Grando e Prado. Para tal, utilizou-se a metodologia Box-Jenkins como forma de descrever a sazonalidade para o problema em estudo. Os dados, publicados por Grando e Prado, sobre volume mensal de água consumida no período compreendido entre outubro de 2010 e setembro de 2014 em uma cidade do oeste de Santa Catarina, foram utilizados para a modelagem do problema a partir da seguinte metodologia: Identificação do problema; Estimação dos parâmetros e teste \& Aplicação. Para tornar a série estacionária foram realizadas uma primeira diferenciação e uma diferenciação sazonal. A partir da análise das Funções de Autocorrelação e Autocorrelação Parcial da série devidamente estacionária foram estimados 04 modelos, sendo escolhido como mais adaptado, a partir dos critérios de informação AIC e BIC, o modelo SARIMA $(1,2,1)(1,1,0) 12$. Em comparação com o modelo proposto por Grando e Prado, constatou-se uma redução de 23 \%, 22 \% e 37 \% nos erros MAPE, MAD e EQM. Portanto, a utilização de modelos matemáticos se mostra como uma alternativa bastante viável na gestão de recursos hídricos, uma vez que podem ser empregados para prever, analisar e auxiliar os processos de tomada decisão referentes à utilização e preservação de tais recursos.

Palavras-chave: Modelos de Previsão; Recursos Hídricos; Sarima.

\section{Mathematical models of forecasting applied to water management}

\begin{abstract}
The advance of urbanization and the absence of effective public policies are presented as challenges to the proper provision of water supply services, however, in Brazil, the raising awareness about the importance of preserving water resources finds as barrier its abundance. An alternative to assist water resource management activities is the use of mathematical models. This study aims to develop a mathematical model for predicting the water consumption building on the proposed by Grando and Prado. For such, was utilized the methodology of BoxJenkins as a way to describe seasonality to the problem under study. The data, published by Grando and Prado, about monthly volume of water consumed in the period between october 2010 and september 2014 in a town located in west of Santa Catarina state, was used for the modeling of the problem with the following methodology: Problem Identification; Parameter estimation and test \& Application. To make the series stationary were carried out a first difference and a seasonal difference. From the analysis of the Autocorrelation and Autocorrelation and Partial Functions properly stationary series were estimated 04 models, being chosen as most appropriate, from the AIC and BIC Information Criterions, the model SARIMA $(1,2,1)(1,1,0) 12$. Compared to the model proposed by Grando and Prado, were found reductions of $23 \%, 22 \%$ and $37 \%$ in the MAPE, MAD and EQM errors. Therefore, the use of mathematical models are a viable alternative in water management, since it can be used to predict, analyze and auxiliary the decision making processes regarding the use and conservation of such resources.
\end{abstract}

Keywords: Forecasting Models; Water Resources; Sarima.

Topic: Engenharia de Recursos Hídricos

Reviewed anonymously in the process of blind peer

Jair Paulino de Sales

Universidade Federal do Cariri, Brasil

http://lattes.cnpq.br/7099203116962634

jairpsales@hotmail.com

João Marcos Pereira Silva

Universidade Federal da Paraíba, Brasil

http://lattes.cnpq.br/5631140865699694

m.joaop@hotmail.com

Andersson Alves da Silva

Universidade Regional do Cariri, Brasil

http://lattes.cnpq.br/7607260025203848

andersson.chester@gmail.com

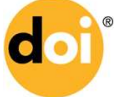

DOI: 10.6008/SPC2179-6858.2016.003.0008
Received: 20/03/2016

Approved: 10/08/2016

\author{
Jackson de Sousa Silva \\ Universidade Regional do Cariri, Brasil \\ http://lattes.cnpq.br/3276775491060001 \\ jacksonsssousa@hotmail.com
}

Referencing this:

SALES, J. P.; SILVA, J. M. P.; SILVA, A. A.; SILVA, J. S.. Modelos matemáticos de previsão aplicados à gestão hídrica. Revista IberoAmericana de Ciências Ambientais, v.7, n.3, p.90-99, 2016. DOI: http://doi.org/10.6008/SPC2179-6858.2016.003.0008 


\section{INTRODUÇÃO}

O entendimento sobre o valor da água é indispensável para o desenvolvimento de qualquer civilização, e este se justifica por uma causa majoritária: “a água é essencial à vida” (PATO, 2007). Entretanto, no Brasil, a tomada de consciência sobre a relevância da preservação de tal recurso encontra como barreira a abundância do mesmo (IORIS, 2009).

Desde a colonização brasileira os rios foram utilizados como rotas de transporte e acesso ao interior, portanto as reservas hídricas influenciaram diretamente na alocação dos primeiros núcleos urbanos (ANA, 2007; IORIS, 2009).

O avanço da urbanização e a ausência de políticas públicas eficazes, hoje, apresentam-se como desafios para a disposição adequada dos serviços de abastecimento hídrico (IORIS, 2009). Inicialmente estes problemas surgiram na Europa do século XIX, centro do desenvolvimento mundial da época, e posteriormente nas demais regiões em crescimento. Logo, houve a necessidade de se criarem serviços de abastecimento e saneamento capazes de garantir que a água chegasse em quantidade e qualidade suficientes aos consumidores (PATO, 2007). Apenas a partir da década 1990 o setor industrial passa a inteirarse a respeito de sua participação nas discussões sobre a água, sendo esta considerada em 2014, no Fórum Econômico Mundial, o terceiro mais relevante entre os dez maiores riscos globais (MARTINS, 2014).

Hoesktra (2012) apresenta dados afirmando que algumas regiões do mundo atualmente registram quadros de insuficiência hídrica, e nestes, em alguns períodos do ano, a quantidade de água disponível é menor do que a necessária para o consumo, afetando aproximadamente dois bilhões de pessoas, e esta situação se agrava quando consideradas as mudanças climáticas decorrentes do aquecimento global, sendo este causado pelo homem ou mesmo um evento natural (MARTINS, 2014).

Para Luchini (2000), nos últimos anos o desenvolvimento no campo da biologia e da teoria geral dos sistemas (BERTALANFFY, 2010) vem proporcionando novos conceitos que possibilitam um comportamento interdisciplinar e multifocado, diferente da abordagem tradicional. Uma alternativa para auxiliar atividades de gestão dos recursos hídricos é a utilização de modelos matemáticos, e há uma diversidade destes. Podem ser utilizados para prever, analisar e auxiliar processos de tomada de decisões, entretanto para que sejam confiáveis se faz necessária uma abordagem de minimização de erros. Usar a modelagem matemática para contribuir na solução de problemas oriundos dos impactos ambientais tem se tornado vantajoso, tanto do ponto de vista econômico ou ambiental.

Segundo Rosa et al. (2012), o uso da modelagem matemática para fenômenos ambientais começou a ser estudado e desenvolvido no início do século XX a partir de diversos trabalhos de Streeter e Phelps que construíram um modelo capaz de analisar o perfil de oxigênio dissolvido, e, assim, contribuiu com a previsão do comportamento de poluentes em solos, águas e atmosfera.

Partindo para o contexto atual, prever os níveis críticos que provocam a escassez dos recursos hídricos, assim como os desgastes aos componentes naturais, permitem que o ser humano atue através da 
melhor tomada de decisão para combater o desperdício, a má utilização e propor um novo gerenciamento de tal recurso.

Existem diversos tipos de modelos matemáticos utilizados na gestão ambiental, sendo os mais complexos e dinâmicos desenvolvidos pela Companhia Ambiental do Estado de São Paulo (CETESB) e pela Agência de Proteção Ambiental dos Estados Unidos (US-EPA), que são usados para melhorar o entendimento e capacidade de análise dos sistemas naturais que auxiliem na tomada de decisão relacionadas ao meio ambiente, assim como a fiscalização do uso dos recursos (ROSA et al., 2012).

Feil et al. (2013) realizaram estudos sobre a previsão do consumo de água na cidade de Lajeado no Rio Grande do Sul. O estudo foi possível a partir da modelagem matemática aplicado ao sistema de abastecimento de água local, que previu o consumo de água da cidade entre os anos de 2008 e 2032. Os resultados auxiliaram a Companhia Riograndense de Saneamento (CORSAN) no gerenciamento da demanda de água do município, assim como na elaboração de uma linha de tendência que permitiu prever a partir do aumento populacional uma eventual falta de água no abastecimento.

Grando et al. (2015) elaboraram um modelo de previsão a partir de uma regressão linear utilizando dados históricos de 48 meses (outubro de 2010 a setembro de 2014) como tentativa de dar suporte ao problema de falta de água potável em períodos de estiagem no estado de Santa Catarina.

Doravante tais conceitos, este trabalho tem por objetivo criar um modelo matemático de previsão do consumo hídrico mais assertivo do que o elaborado por Grando et al. (2015). Para tal, utilizar-se-á a metodologia Box-Jenkins como forma de descrever a sazonalidade para o problema em estudo.

\section{REVISÃO TEÓRICA}

\section{Limitações nos Modelos de Regressão Linear}

Escolher a técnica de previsão mais assertiva a partir de séries temporais é essencial para se chegar ao melhor modelo, e este será mais adaptado ao fenômeno em estudo quando for capaz de descrever o comportamento sazonal, caso exista, da série temporal em análise.

Entende-se por série temporal um grupo de observações ordenadas e espaçadas em um mesmo intervalo de tempo (WERNER et al., 2003). Entre as características que definem uma série temporal destacase a presença, ou não, de tendência e sazonalidade, entre a quais a primeira refere-se ao sentido do deslocamento adotado pela série no decorrer do período analisado, e a segunda diz respeito ao movimento ondulatório da série em intervalos de tempos analisados.

Presumindo uma maior confiabilidade na previsão, a regressão linear é limitada, pois, na sua elaboração não são consideradas as variações aleatórias e de sazonalidade, além de estarem predestinadas a apresentarem tendências crescentes ou decrescentes em períodos curtos dentro de uma série mais extensa (PEINADO et al., 2007). Portanto, para que o modelo a ser elaborado seja capaz de descrever o comportamento do fenômeno em estudo de forma eficiente faz-se necessária a utilização de metodologias capazes de descrever o comportamento sazonal da série. 
O uso do método de regressão linear na previsão do modelo de Grando et al. (2015), embora bastante promissor, falha nesse quesito. O histórico de água potável segue uma pequena sazonalidade, que não foi percebida pela regressão linear aplicada. Sendo assim, diante dos argumentos apresentados, podese afirmar que, como a série temporal do histórico da água potável na região oeste de Santa Catarina possui índices de sazonalidade, conseguir-se-á uma melhor previsão considerando as variações sazonais destacadas na série em estudo.

\section{Metodoliga Box-Jenkins}

Os modelos Box-Jenkins, também conhecidos como um conjunto de processos estocásticos chamados de ARIMA (Auto regressivo Integrado e de Médias Móveis), têm como objetivo realizar previsões a partir de séries temporais, verificando e determinando três componentes não sazonais: parâmetros autoregressivos $A R(p)$, processos de integração (diferenciação) I(d) e parâmetros de médias móveis $M A(q)$. $A$ metodologia de Box-Jenkins pode descrever tanto o comportamento de séries estacionárias como o de não estacionárias (AMORIM, 2012). A aplicabilidade do emprego da metodologia devido à confiabilidade dos seus resultados prosperou, havendo um aumento considerável no número de trabalhos publicados.

A partir da necessidade da adição do comportamento sazonal aos modelos de previsão surgiram os modelos SARIMA (Seasonal Autoregressive Integrated Moving Average). Estes modelos apresentam tanto uma parte não sazonal quando uma sazonal, descrita pelos parâmetros $A R(P)$, I(D) e $M A(Q)$. Werner e Ribeiro (2003) apresenta a equação 01 como modelo geral.

$$
\begin{gathered}
\left(1-\emptyset_{1} L-\cdots-\emptyset_{p} L^{p}\right)\left(1-\Phi_{1} L^{S}-\cdots-\Phi_{P} L^{P S}\right)(1-L)^{d}\left(1-L^{S}\right)^{D} Z_{t}= \\
\left(1-\theta_{1} L-\cdots-\theta_{q} L^{q}\right)\left(1-\Theta_{1} L-\cdots-\Theta_{Q} L^{Q S}\right) \varepsilon_{t}
\end{gathered}
$$

Onde:

$\left(1-\emptyset_{1} L-\cdots-\emptyset_{p} L^{p}\right)=$ componente autoregressivo e não-sazonal de ordem $\mathrm{p} ;$ $\left(1-\Phi_{1} L^{S}-\cdots-\Phi_{P} L^{P S}\right)=$ componente autoregressivo e sazonal de ordem $\mathrm{P}$ e fator de sazonalidade $\mathrm{S}$; $(1-L)^{d}=$ componente de integração não sazonal de ordem d; $\left(1-L^{S}\right)^{D}=$ componente de integração sazonal de ordem $\mathrm{D}$ e fator de sazonalidade $\mathrm{S}$;

$\left(1-\theta_{1} L-\cdots-\theta_{q} L^{q}\right)=$ componente de médias móveis não sazonal de ordem q; $\left(1-\Theta_{1} L-\cdots-\Theta_{Q} L^{Q S}\right)=$ componente de médias móveis sazonal de ordem $Q$ e fator de sazonalidade $\mathrm{S}$.

\section{Critérios de Avaliação dos Modelos De Previsão}

Peinado et al. (2007) afirmam que os modelos de decomposição de séries temporais não podem ser considerados infalíveis, ou seja, sem considerar os erros, muito embora possuam alto grau de assertividade perante as previsões. Para uma melhor visão e análise dos dados futuros, recomenda-se utilizar vários métodos diferentes e compará-los com o valor real.

Em decorrência do comportamento da série temporal que se deseja analisar, vários modelos podem ser utilizados na previsão de seus valores futuros. A escolha do modelo mais adequado é feita, geralmente, a partir do somatório dos erros gerados por cada modelo (PELLEGRINI, 2000). O erro de previsão consiste na diferença entre o valor real e o valor previsto pelo modelo para dado período da série (TUBINO, 2000). 
Uma vez que o modelo de previsão pode gerar, aleatoriamente, valores acima e abaixo dos reais, o que consequentemente resultará em valores de erros positivos e negativos, espera-se que o erro acumulado tenda a zero (PELLEGRINI, 2000; TUBINO, 2000). Diversas formas de cálculo para o somatório dos erros podem ser utilizadas, constituindo-se, assim como critérios para escolha de modelos mais confiáveis.

Dentre os critérios mais utilizados para avaliar a precisão de um modelo quantitativo de previsão podemos listar: Desvio absoluto médio (Mean Absolute Deviation - MAD), Erro Percentual Absoluto Médio (Mean Absolute Percentual Error - MAPE) e Erro Quadrado Médio (EQM).

Utiliza-se o MAD para avaliar o nível de erro na mesma unidade dos dados (Equação 02), o MAPE para avaliar a magnitude do erro com relação à série (Equação 03), já o EQM é o valor dos erros ao quadrado (Equação 04). O modelo apropriado será aquele que obtiver o menor erro no critério de cálculo escolhido.

$$
\begin{gathered}
M A D=\frac{1}{n} \sum_{t=1}^{n}\left|e_{t}\right| \\
M A P E=\frac{1}{n} \sum_{t=1}^{n}\left|\frac{e_{t}}{z_{t}} \times 100\right| \\
E Q M=\frac{1}{n} \sum_{t=1}^{n} e_{t}^{2}
\end{gathered}
$$

Nas equações, $n$ corresponde ao número de observações, $e_{t}$ é o erro de previsão no período t e $z_{t}$ é o valor real da série no período t.

\section{METODOLOGIA}

Os dados publicados por Grando et al. (2015) sobre volume mensal de água consumida no período compreendido entre outubro de 2010 e setembro de 2014 em uma cidade do oeste de Santa Catarina serviram de base para a elaboração de 04 modelos a partir da metodologia descrita por Sato (2013): Identificação; Estimação e teste \& Aplicação.

Identificação: verificou-se a existência de padrões como sazonalidade e ciclos na série em estudo a partir da utilização das funções de autocorrelação (FAC) e autocorrelação parcial (FACP). Tanto a FAC quanto a FACP objetivam a percepção sobre a existência de correlação entre os episódios do fenômeno em análise. Caso a série seja não estacionária a FAC apresentará resultados estatisticamente significativos, demonstrando, em seu gráfico, comportamento senoidal amortecido ou lento decaimento. Para tornar a série estacionária é necessária a execução de diferenciações. Caso a série apresente sazonalidade executa-se a diferença sazonal. Para confirmar a estacionariedade da série optou-se pela realização do teste da raiz unitária de Dickey-Fuller Aumentado a partir do software EViews 7.0.

Estimação e teste: Foram determinados os parâmetros $A R(p), A R(P), M A(q)$ e $M A(Q)$, todos estatisticamente significativos $(p$-valor $<0.05)$. Normalmente mais de um modelo pode ser encontrado, portanto faz necessária a utilização de critérios de comparação para a escolha do 
modelo mais adequado. Foram utilizados os Critérios de Informação de Schwartz (BIC) e Akaike (AIC) descritos, respectivamente, pelas equações 05, 06. Após identificação do melhor modelo verificouse o comportamento de seus resíduos a partir das FAC e FACP residuais. Considerou-se como melhor modelo aquele que apresentou menores valores para o BIC, AIC e EQM.

$$
\begin{gathered}
B I C=\ln \sigma_{\varepsilon}^{2}+\frac{(p+q) \ln (n)}{n} \\
A I C=\ln \sigma_{\varepsilon}^{2}+\frac{2(p+q)}{n}
\end{gathered}
$$

Para as quais $n$ representa o número de observações, $e_{t}$ o resíduo (diferença entre o valor real e o previsto) e $\sigma_{\varepsilon}^{2}$ a variância dos resíduos.

Aplicação: Após escolha do melhor modelo realizou-se a previsão para o período em estudo. Salienta-se que tanto os cálculos dos critérios de informação, das FAC e FACP e a execução da previsão foram realizados a partir dos softwares Gretl 1.10.2 e Microsoft Excel 2010.

Após escolha do modelo e realização da previsão foram calculados os MAD, MAPE e EQM, tanto do modelo proposto por Grando e Prado (2015) quanto do modelo aqui elaborado.

\section{RESULTADOS E DISCUSSÃO}

A figura 01 representa o comportamento gráfico da série estudada por Grando et al. (2015), e a partir de sua análise percebeu-se um comportamento não estacionário. Para a confirmação de sua não estacionariedade realizou-se, também, análise em suas FAC e FACP (Figura 02), demonstrando um comportamento senoidal amortecido, outro indicativo de não estacionariedade. Como critério quantitativo de confirmação realizou-se o teste de Dickey-Fuller Aumentado. Como $\tau$ é maior que o valor crítico ao nível de $5 \%$ a hipótese nula de raiz unitária não é rejeitada, logo a série é não estacionária $(\tau=-2,216405>$ $-2,926622)$.

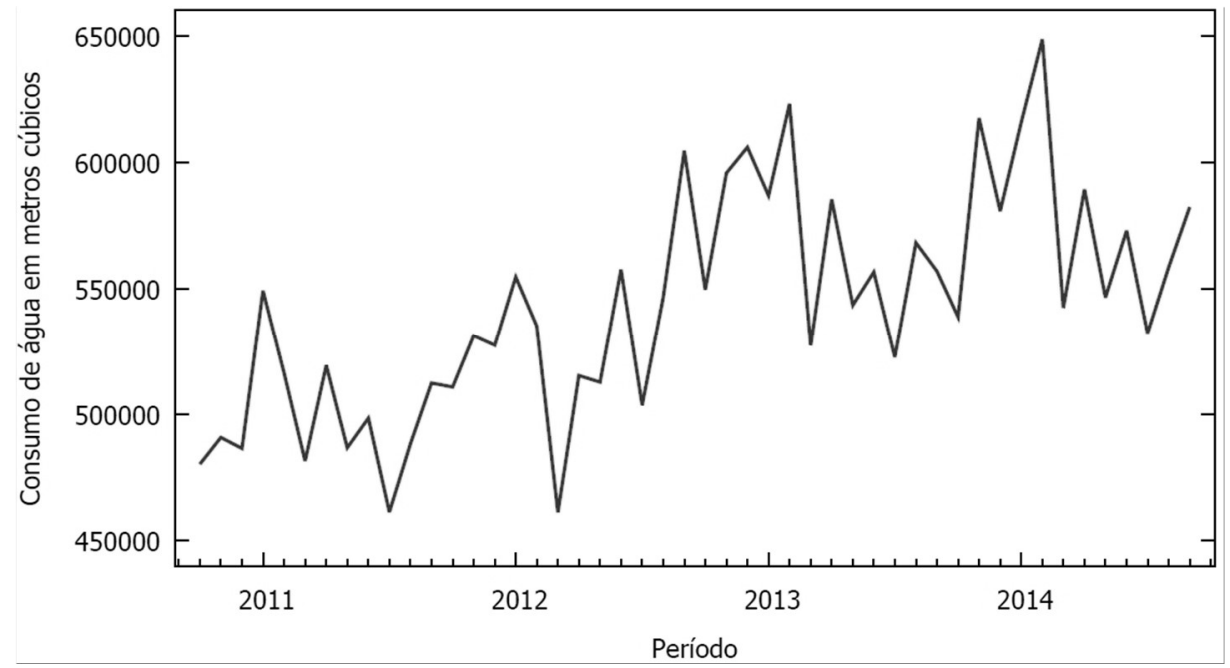

Figura 01: Consumo de água em metros cúbicos. Fonte: Grando et al. (2015). 


\begin{tabular}{|c|c|c|c|c|c|c|c|c|}
\hline \multicolumn{2}{|c|}{ FAC } & \multicolumn{3}{|c|}{ FACP } & FAC & FACP & Q-Stat & Prob \\
\hline 1 & $\square$ & 1 & $\square$ & 1 & 0.511 & 0.511 & 13.352 & 0.000 \\
\hline 1 & $\square$ & 1 & $\square$ & 2 & 0.564 & 0.409 & 29.930 & 0.000 \\
\hline 1 & $\square$ & 1 & घ & 3 & 0.462 & 0.134 & 41.319 & 0.000 \\
\hline 1 & $\square$ & & 1 & 4 & 0.345 & -0.076 & 47.814 & 0.000 \\
\hline 1 & $\square$ & 1 & 1 & 5 & 0.363 & 0.073 & 55.178 & 0.000 \\
\hline 1 & ? & '맘 & 1 & 6 & 0.126 & -0.241 & 56.081 & 0.000 \\
\hline 1 & $\square$ & 15 & $\nabla$ I & 7 & 0.301 & 0.228 & 61.384 & 0.000 \\
\hline 1 & $\square$ & 1 & 曰 & 8 & 0.241 & 0.160 & 64.857 & 0.000 \\
\hline 1 & $\square$ & 1 & 1 & 9 & 0.223 & 0.009 & 67.910 & 0.000 \\
\hline 1 & $\square$ & 1 & 1 & 10 & 0.245 & -0.023 & 71.712 & 0.000 \\
\hline 1 & 21 & 1 도 & 1 & 11 & 0.114 & -0.146 & 72.559 & 0.000 \\
\hline 1 & $\square$ & 1 & $\square$ & 12 & 0.356 & 0.290 & 81.009 & 0.000 \\
\hline 1 & 21 & '맘 & 1 & 13 & 0.071 & -0.208 & 81.355 & 0.000 \\
\hline 1 & 曰 & 11 & 1 & 14 & 0.138 & -0.036 & 82.702 & 0.000 \\
\hline 1 & 1 & 1 든 & 1 & 15 & 0.049 & -0.152 & 82.878 & 0.000 \\
\hline 1 & 1 & 1 & 1 & 16 & 0.013 & 0.030 & 82.889 & 0.000 \\
\hline 1 & 1 & 1 & 1 & 17 & 0.042 & -0.040 & 83.024 & 0.000 \\
\hline $1 \square$ & 1 & I마 & 1 & 18 & -0.213 & -0.189 & 86.647 & 0.000 \\
\hline 14 & 1 & 1 도 & 1 & 19 & -0.113 & -0.147 & 87.699 & 0.000 \\
\hline $1 \square$ & 1 & 1 & 1 & 20 & -0.174 & 0.003 & 90.283 & 0.000 \\
\hline
\end{tabular}

Figura 02: Correlograma da FAC para o consumo de água.

Com o objetivo de estacionarizar a série realizou-se uma primeira diferenciação, e, para esta, o teste de Dickey-Fuller Aumentado obteve $\tau$ menor que o valor crítico ao nível de $5 \%(\tau=-12,76318<$ -2,926622), portanto a hipótese nula de raiz unitária é rejeitada admitindo a primeira diferença como estacionária. A figura 03 mostra o correlograma da série com uma diferenciação. Desta forma, adotou-se $I(d)=1$

\begin{tabular}{|c|c|c|c|c|c|c|c|c|}
\hline \multicolumn{2}{|c|}{ FAC } & \multicolumn{2}{|c|}{ FACP } & & FAC & FACP & Q-Stat & Prob \\
\hline ᄃ & 1 & & 1 & 1 & -0.574 & -0.574 & 16.483 & 0.000 \\
\hline 1 & $\square$ ○ & $1 \square$ & 1 & 2 & 0.176 & -0.229 & 18.060 & 0.000 \\
\hline & 1 & 10 & 1 & 3 & 0.044 & 0.057 & 18.161 & 0.000 \\
\hline & 1 & 10 & 1 & 4 & -0.161 & -0.102 & 19.547 & 0.001 \\
\hline 1 & $\square$ & 19 & 1 & 5 & 0.231 & 0.117 & 22.471 & 0.000 \\
\hline$\square$ & 1 & $\square$ & 1 & 6 & -0.402 & -0.338 & 31.558 & 0.000 \\
\hline & $\square$ । & $\square$ & 1 & 7 & 0.180 & -0.354 & 33.428 & 0.000 \\
\hline 1 & 1 & 1마 & 1 & 8 & -0.026 & -0.205 & 33.468 & 0.000 \\
\hline & 1 & 10 & 1 & 9 & -0.059 & -0.098 & 33.681 & 0.000 \\
\hline & 口 & 10 & 1 & 10 & 0.167 & 0.093 & 35.420 & 0.000 \\
\hline$\square$ & 1 & $\square$ & 1 & 11 & -0.355 & -0.397 & 43.465 & 0.000 \\
\hline 1 & & 1 & 1 & 12 & 0.564 & 0.104 & 64.379 & 0.000 \\
\hline & 1 & 15 & 1 & 13 & -0.376 & -0.063 & 73.960 & 0.000 \\
\hline 1 & $\square$ & & 1 & 14 & 0.188 & 0.114 & 76.421 & 0.000 \\
\hline 1 & 1 & 10 & 1 & 15 & -0.054 & -0.107 & 76.627 & 0.000 \\
\hline & 1 & 10 & 1 & 16 & -0.081 & -0.073 & 77.116 & 0.000 \\
\hline 1 & $\square$ & 1 & 1 & 17 & 0.232 & 0.007 & 81.238 & 0.000 \\
\hline$\square$ & 1 & 1 & 1 & 18 & -0.322 & 0.087 & 89.468 & 0.000 \\
\hline 1 & . & 1 & 1 & 19 & 0.126 & -0.048 & 90.780 & 0.000 \\
\hline 1 & 1 & 1 & 1 & 20 & 0.035 & 0.031 & 90.885 & 0.000 \\
\hline
\end{tabular}

Figura 03: Correlograma da FAC para a série diferenciada uma vez.

Para identificação dos possíveis modelos os parâmetros $A R(p), M A(q), A R(P)$ e $M A(Q)$ foram selecionados a partir das FAC e FACP da série devidamente estacionarizada. Para eliminação da influência 
sazonal $(s=12)$ na série realizou-se uma diferença sazonal, logo o parâmetro $I(D)=1$. Os modelos propostos são apresentados na tabela 01 .

Tabela 01: Modelos propostos.

\begin{tabular}{|c|c|c|c|c|}
\hline Modelo & Coeficientes & $p$-valor dos coeficientes & AIC & BIC \\
\hline SARIMA $(2,1,0)(1,1,0)_{12}$ & $\begin{array}{l}\emptyset_{1}=-0,599189 \\
\emptyset_{2}=-0,330686 \\
\Phi_{1}=-0,381531\end{array}$ & $\begin{array}{l}<0,0002 \\
<0,0347 \\
<0,0223\end{array}$ & 822,62 & 816,40 \\
\hline SARIMA $(2,1,0)(0,1,0)_{12}$ & $\begin{array}{l}\emptyset_{1}=-0,546092 \\
\emptyset_{2}=-0,344526\end{array}$ & $\begin{array}{l}<0,0006 \\
<0,0268\end{array}$ & 818,74 & 823,40 \\
\hline SARIMA $(1,1,0)(1,1,0)_{12}$ & $\begin{array}{l}\emptyset_{1}=-0,446294 \\
\Phi_{1}=-0,398443\end{array}$ & $\begin{array}{l}<0,0029 \\
<0,0162\end{array}$ & 818,53 & 823,20 \\
\hline SARIMA $(1,2,1)(1,1,0)_{12}$ & $\begin{array}{c}\emptyset_{1}=-0,435561 \\
\theta_{1}=-0,392076 \\
\Phi_{1}=-0,999999\end{array}$ & $\begin{array}{l}<0,0045 \\
<0,0224 \\
<0,0000\end{array}$ & 802,83 & 808,94 \\
\hline
\end{tabular}

A partir dos critérios de informação AIC e BIC o modelo SARIMA $(1,2,1)(1,1,0)_{12}$ foi selecionado como o mais assertivo. Salienta-se que para os modelos estatisticamente significativos todos os coeficientes devem possuir $p$-valor $<0.05$, o que de fato ocorreu com o selecionado. Para confirmação de sua adequação à série em estudo optou-se pela realização de um teste de normalidade para os seus resíduos (Figura 04). Os resíduos apresentaram homocedasticidade, logo são favoráveis à adoção do modelo.

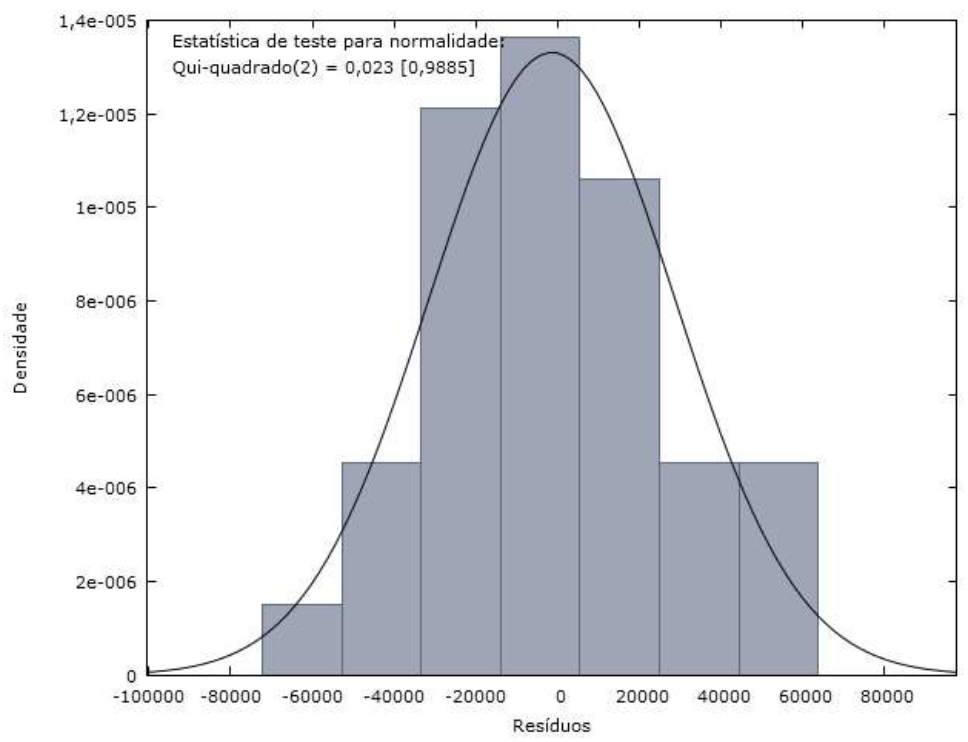

Figura 04: Distribuição normal dos resíduos.

A partir do modelo SARIMA $(1,2,1)(1,1,0)_{12}$ realizou-se a previsão para os próximos doze meses do consumo de água na cidade em estudo. A tabela 02 compara os dados a partir dos MAPE, MAD e EQM do modelo de regressão linear proposto por Grando e Prado (2015) e o aqui elaborado. Houve redução de respectivamente $23 \%, 22 \%$ e $37 \%$ nos erros.

Tabela 02: Comparação entre erros dos modelos.

\begin{tabular}{c|c|c|c}
\hline MODELO & MAPE & MAD & EQM \\
\hline Grando e Prado $(2015)$ & 5,26 & 29654,47 & $1.308 .722 .773,80$ \\
\hline SARIMA $(1,2,1)(1,1,0)_{12}$ & 4,07 & 22983,78 & $820.727 .910,81$ \\
\hline Redução no erro & $23 \%$ & $22 \%$ & $37 \%$ \\
\hline
\end{tabular}


A figura 05 demonstra o comportamento da série, assim como o bom desempenho do modelo proposto com seu intervalo de confiança. O modelo foi capaz de descrever a sazonalidade da série. A tabela 03 dispõe a comparação entre os valores do modelo SARIMA $(1,2,1)(1,1,0)_{12}$ e do proposto por Grando et al. (2015).

Tabela 03. Comparação entre os valores das previsões dos modelos.

\begin{tabular}{c|c|c}
\hline Modelo & SARIMA (1,2,1)(1,1,0) $\mathbf{1 2}$ & Grando e Prado (2015) \\
\hline Outubro - 2014 & 543890,55 & 595020,93 \\
\hline Novembro - 2014 & 609748,49 & 597126,75 \\
\hline Dezembro - 2014 & 588867,68 & 599232,56 \\
\hline Janeiro - 2015 & 601151,66 & 601338,38 \\
\hline Fevereiro - 2015 & 633454,34 & 603444,20 \\
\hline Março - 2015 & 529741,39 & 605550,02 \\
\hline Abril - 2015 & 579094,16 & 607655,84 \\
\hline Maio - 2015 & 534955,30 & 609761,65 \\
\hline Junho - 2015 & 554418,38 & 611867,47 \\
\hline Julho - 2015 & 514635,28 & 613973,29 \\
\hline Agosto - 2015 & 546597,32 & 616079,10 \\
\hline Setembro - 2015 & 554822,78 & 618184,92
\end{tabular}

Entre os valores propostos por ambos os modelos se percebe uma disparidade maior a partir de março de 2015, período de queda das temperaturas na região devido à chegado do outono.

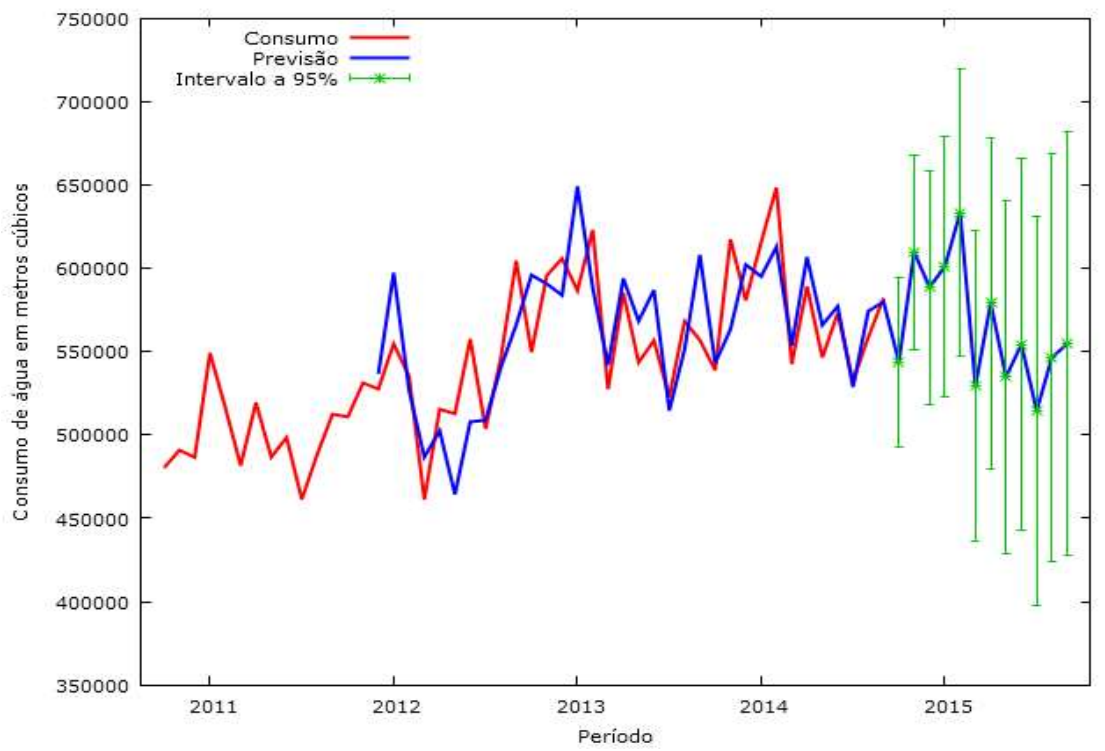

Figura 05: Série original, modelo proposto e intervalo de confiança.

\section{CONSIDERAÇÕES FINAIS}

A utilização de modelos matemáticos se mostra como uma alternativa bastante viável na gestão de recursos hídricos, uma vez que podem ser empregados para prever, analisar e auxiliar os processos de tomada decisão referentes à utilização e preservação de tais recursos.

É relevante salientar que quando se utilizam modelos de previsão, em meio aos diversos existentes, deve-se escolher aquele que possuir resultados mais assertivos, podendo descrever a série temporal em 
estudo com uma maior acuracidade. Para este fim é recomendável a utilização de critérios de avaliação dos modelos de previsão como aqueles citados neste trabalho.

Através dos resultados obtidos percebeu-se que o modelo de previsão proposto, SARIMA $(1,2,1)(1,1,0)_{12}$, mostra melhores resultados perante o modelo de regressão linear proposto por Grando et al. (2015), apresentando uma melhoria de $23 \%, 22 \%$ e $37 \%$ nos critérios MAPE, MAD e EQM, respectivamente. Tal aperfeiçoamento pode ser atribuído ao fato de que, diferentemente da regressão linear, o modelo SARIMA considera os componentes de sazonalidade presentes na série temporal, aumentando a acuracidade da previsão.

\section{REFERÊNCIAS}

AMORIM, M. R. B.. Modelagem em séries temporais aplicado a dados de temperatura máxima na cidade de Campina Grande - PB. Trabalho de Conclusão de Curso (Graduação em Estatística) - Universidade Estadual da Paraíba, Campina Grande, 2012.

ANA. A história do uso da água no Brasil. Do descobrimento ao século XX. Brasília: ANA, 2007.

BERTALANFFY, L. V.. Teoria geral dos sistemas. 5 ed. Petropolis: Vozes, 2010.

FEIL, A. A.; HAETINGER, C.. Previsão de consumo de água via modelagem matemática de sistema de abastecimento de água. Revista DAE, n.195, p.32-46, 2013. DOI:

http://dx.doi.org/10.4322/dae.2014.119

GRANDO, M. L.; PRADO, R.. Estudo de previsão de demanda de água potável de um município do oeste do estado de Santa Catarina. Tecnológica, v.3, n.2, p.246-258, 2015.

HOEKSTRA, A. Y.; MEKONNEN, M. M.; CHAPAGAIN, A. K.; MATHEWS, R. E.; RICHTER, B. D.. Global Monthly Water Scarcity: Blue Water Footprints versus Blue Water Availability. PLoS ONE, v.7, n.2, 2012. DOI: http://dx.doi.org/10.1371/journal.pone.0032688

IORIS, A.. Desenvolvimento nacional e gestão de recursos hídricos no Brasil. Revista Crítica de Ciências Sociais, v.85, p.23-41, 2009. DOI: http://dx.doi.org/10.4000/rccs.329

LUCHINI, A. M.. Os desafios à implementação do sistema de gestão dos recursos hídricos estabelecido pela Lei $n$. 9.433/97. RAP, v.34, n.1, p.123-143, 2000.
MARTINS, R. S. L.. Empresas e gestão da água: uma abordagem a partir do uso indicador pegada hídrica. Tese (Doutorado em Ciências Ambientais) - Universidade de São Paulo, São Paulo, 2014.

PATO, J. H.. O valor da água como bem público. Tese (Doutorado em Sociologia) - Universidade de Lisboa, Lisboa, 2007.

PEINADO, J.; GRAEML, A. R.. Administração da Produção: operações industriais e serviços. Curitiba: Unicenp, 2007.

PELLEGRINI, F. R.. Metodologia para implementação de sistemas de previsão de demanda. Dissertação (Mestrado em Engenharia de Produção) - Universidade Federal Rural do Rio Grande do Sul, Porto Alegre, 2000.

ROSA, A. H.; FRACETO. L. F.; MOSCHINI-CARLOS, V. (Org.). Meio Ambiente e Sustentabilidade. Porto Alegre: Bookman, 2012.

SATO, R. C. Gerenciamento de doenças utilizando séries temporais com o modelo ARIMA. Einstein, v.11, n.1, p.128131, 2013. DOI: http://dx.doi.org/10.1590/S1679$\underline{45082013000100024}$

TUBINO, D. F. Manual de Planejamento e Controle da Produção. 2 ed. São Paulo: Atlas, 2000.

WERNER, L.; RIBEIRO, J. L. D.. Previsão de demanda: uma aplicação dos modelos Box-Jenkins na área de assistência técnica de computadores pessoais. Gestão e Produção, v.10, n.1, p.47-67, 2003. DOI: http://dx.doi.org/10.1590/S0104$\underline{530 \times 2003000100005}$ 